\title{
Evaluation of the case mix and equity of age-sex adjusted primary care capitation payment models in Ontario, Canada Lyn M Sibley*1 and Richard H Glazier ${ }^{2}$
}

\begin{abstract}
Address: ${ }^{1}$ Health System Performance Research Network, Department of Health Policy, Management, and Evaluation, University of Toronto, Canada and ${ }^{2}$ Centre for Research on Inner City Health and Department of Family and Community Medicine, St. Michael's Hospital, Institute for Clinical Evaluative Sciences (ICES), Departments of Family and Community Medicine and Dalla Lana School of Public Health, University of Toronto, Canada

* Corresponding author
\end{abstract}

from 25th Patient Classification Systems International (PCSI) Working Conference

Fukuoka, Japan. II-I4 November 2009

Published: 5 November 2009

BMC Health Services Research 2009, 9(Suppl I):A12 doi:I0.1I86/I472-6963-9-SI-AI2

This abstract is available from: http://www.biomedcentral.com/I472-6963/9/SI/AI2

(c) 2009 Sibley and Glazier; licensee BioMed Central Ltd.

\section{Introduction}

Several innovative models for primary care delivery have recently been introduced in Ontario, Canada. These group-practice models share common characteristics of patient rostering, age-sex based capitation rates, and performance-based incentives. These models have been adopted because of their potential strengths to reduce the overall cost of care, improve effective medical care management, and increase the use of appropriate preventativecare measures. There is concern, however, that age-sex adjusted capitation rates alone do not take into account variations in the morbidity burden and the healthcare needs that are associated with socioeconomic status.

The objective of this study is to compare capitation remuneration rates, by socioeconomic status (SES), with the morbidity burden and the expected primary care resource use of patients enrolled to primary care physicians in the most established capitation model -- Family Health Networks (FHN).

\section{Results}

The study sample consisted of 487,131 patients enrolled to 507 physicians in 53 group practices continuously from September 1, 2005 to August 31, 2006. The number of ADGs (types of diagnosed conditions) and ACG weights (relative expected resource use) was higher among women, and increased incrementally with increasing age. This finding was consistent across income quintiles.
Low SES was associated with a higher average number of ADGs compared to the highest SES (2.7 vs 2.9; $\mathrm{p}<0.001)$. Average ACG weights were higher in the low SES category compared to the highest $(0.65$ vs. $0.55 ; \mathrm{p}<0.001)$. The average capitation rate increased incrementally with decreasing SES; however, it did not increase at the same rate as the measures of expected resource use. Both the standardized average ADG count, and ACG weight of those in the lowest income group, were higher than the average capitation rate. The opposite was true for those in the highest income group.

\section{Conclusion}

Age-sex adjusted capitation rates do take into account some of the variation in the morbidity burden and the expected healthcare resource utilization that exists across the SES spectrum. However, the physician reimbursement system in FHNs does not take into account all of the variation in morbidity burden that is associated with socioeconomic status. There is a risk that adjusting capitation rates for age and sex alone introduces an incentive to preferentially enrol patients with higher socioeconomic status, or to practice in geographic areas where residents have higher incomes. 